\title{
ARTICLE \\ Effect of Family Nutrition Therapy on Elderly Patients with Chronic Obstructive Pulmonary Disease
}

\author{
Jun Xue * Yanan Zhang Zhaohe Song Lei Wang Baoyin Bo \\ The Third Affiliated Hospital of Inner Mongolia Medical University, Baotou, Inner Mongolia, 014010, China
}

\section{ARTICLE INFO}

Article history

Received: 30 July 2020

Accepted: 26 August 2020

Published Online: 31 August 2020

Keywords:

Chronic obstructive pulmonary disease

Nutrition support

Elderly

\begin{abstract}
Objective: The aim of the study was to explore the influence of family enteral nutrition support on nutritional status, lung function, activity tolerance and quality of life in elderly patients with chronic obstructive pulmonary disease (COPD) in stable stage and malnutrition. Methods: A prospective observational study of COPD and malnutrition in the geriatric department of our hospital, control group $(\mathrm{n}=82)$ and intervention group $\mathrm{n}=82$ ). The control group was treated with conventional diet, and the intervention group was treated with conventional diet and enteral nutrition suspension. One month later, observe the nutrition indicators,lung function,exercise tolerance and quality of life. Results: (1) BMI and ALB were higher than control group $(\mathrm{t}=10.465,6.189, P<0.05)$, TP and $\mathrm{Hb}$ had no change, $(\mathrm{t}=1.310,1.302, \mathrm{P}>0.05)$. (2) FVC, FEV1, FEV1 FVC, FEV1\% were higher than control group $(\mathrm{t}=11.999,19.654,13.418$, $16.924, P<0.05)$. (3) the quality of life symptom score, activity score, influence score and total score were lower than control group $(\mathrm{t}=15.303$, $6.773,23.600,14.766, P<0.05)$, and 6MWT were higher than control group $(\mathrm{t}=111.962, P<0.05)$. Conclusion:Oral enteral nutritioncan improve the nutritional status, lung function, activity tolerance and quality of life of elderly patients with COPD in stable stage and malnutrition.It can be a early,safe and effective nutritional support strategy for COPD patients with malnutrition
\end{abstract}

2012. Bring a heavy economic burden to society and fami$\operatorname{lies}^{[2]}$. One of the most common respiratory diseases in the elderly is chronic obstructive pulmonary disease (COPD). According to statistics, COPD occupies the fourth place among the causes of death globally, and will jump to the third place by 2020 . The economic burden caused by it will also rank the fifth in the world ${ }^{[3-4]}$. COPD patients with different degrees of malnutrition often affect the respiratory muscle structure and function. The body's defense and resistance functions will also decrease.Pulmo-

*Corresponding Author:

Jun Xue,

The Third Affiliated Hospital of Inner Mongolia Medical University, Baotou, Inner Mongolia, 014010, China;

Email: byxuejun@sina.com 
nary infection is prone to occur. Increasing risk of acute exacerbations of COPD. Our study explored the effects of enteral nutrition on the nutritional status, lung function, activity tolerance and quality of life of elderly patients with COPD and malnutrition.Provide new nutrition methods for elderly patients with COPD and malnutrition.

\section{Methods}

\subsection{Participants}

From April 2016 to April 2018, 165 elderly patients with COPD at stable stage combined with malnutrition were selected as the research objects from the Geriatrics Department of our hospital.

Inclusion criteria: (1) Age of participants was $\geq 65$ years; (2) Diagnostic criteria for COPD reference "global initiative chronic obstructive pulmonary disease (GOLD) 2018 edition" ${ }^{[5]}$. Clinical manifestations of stable COPD ${ }^{[6]}$ : Cough, sputum and shortness of breath are stable or mild. The condition returned to the state before the acute exacerbation. (3) Micro-nutrition assessment method is used for malnutrition standards(MNA-SF), mainly including 6 problems: body mass index decline in 3 months, body mass index, activity ability, stress, neuropsychiatric disease, dietary changes. A total score of 14 points, $\geq 11$ points indicates good nutritional status; 8 -11 points indicates the risk of malnutrition; $<8$ points indicates malnutrition ${ }^{[7]}$. (4) All the candidates can intake half fluid diet through mouth.

Exclusion criteria: (1) Suffer from a disease that cannot eat by mouth, such as gastrointestinal diseases, severe nausea, vomiting, choking on food and water, intestinal obstruction and difficulty swallowing; (2) Patients with severe heart, liver and kidney dysfunction and severe diabetes; (3) Patients who are allergic to any ingredient in enteral nutrition; (4) Patients who did not follow the doctor's instructions to complete the experiment.

This study included 165 participants with COPD and malnutrition. A simple random sampling method is used to divide the selected patients into control group (82 cases) and intervention group ( 83 cases).During the study, there were 3 cases in the control group and 7 cases in the observation group were excluded from this study. 155 participants were included in the final data analysis. There are 79 cases in the control group, including 49 males and 30 females. The average age was $75.6 \pm 3.5$ years old. There are 76 cases in the intervention group, including 40 males and 36 females. The average age was $73.1 \pm 3.1$ years old. All participants were recorded demographic and historical data. There was no significant difference in gender or age between the two groups $(\mathrm{P}>0.05)$.

\subsection{Ethics}

The study was approved by the hospital ethics committee. All participants provided written informed consent.

\subsection{Enteral Nutrition Intervention}

The control group was given a regular diet. The observation group was given enteral nutritional suspension and regular diet. Enteral nutrition is produced by Nutricia Pharmaceutical Co., Ltd ( $1 \mathrm{kcal} / \mathrm{ml}, 500 \mathrm{ml} /$ bottle $)$. All participants take one bottle each time, twice a day. Take $500 \mathrm{ml}$ a day for the first three days. Then gradually increase to $1000 \mathrm{ml}$ according to the patient's tolerance. Intervention time is 1 month. Studies have shown that 1 month of enteral nutrition support can improve lung function in patients with $\mathrm{COPD}{ }^{[8]}$. Participants asked to record their daily diet and enteral nutrient fluid intake. Study staff called participants to verify the details and to seek clarification if required.

\subsection{Lung Function, Exercise Tolerance and Quali- ty of Life Analysis}

Observe the nutritional status of participants after 1 month, including body mass index (BMI), serum albumin (ALB), total protein (TP) and hemoglobin (Hb).

Observe the lung function: Forced vital capacity (FVC), forced expiratory volume in 1 second (FEV1), 1 second rate (FEV1/ FVC), and FEV1 \% of the estimated value. Assessment of activity tolerance: 6 Min Walking Test (6MWT) was used ${ }^{[9]} 6 \mathrm{MWT}$ is suitable for the response of patients with lung disease to medical intervention.6MWT is easy to manage, better tolerated, and better reflects daily activity ability. The technicians conducting the 6MWT test should be trained in the standard scheme.

Assessment of quality of life: St. George's Respiratory Questionnaire (SGRQ) was used. SGRQ was consisted of three points: symptom score, activity score, and score of the influence of disease on patients' daily social activities and psychology. The score was $0-100$, and the lower the score, the higher the quality of life ${ }^{[10]}$. SGRQ is a commonly used questionnaire to evaluate the quality of life of patients. SGRQ is a disease-specific scale. The subjects of this questionnaire include chronic obstructive pulmonary disease and bronchial asthma patients with different countries, races, gender, age, disease severity, and poor lung function. The SGRQ scores are calculated repeatedly. It has been translated into different versions for clinical research needs. SGRQ is suitable for patients with chronic obstructive pulmonary disease worldwide. SGRQ has a good correlation with lung function. It can not only evaluate the treatment of COPD, but also evaluate the overall

DOI: https://doi.org/10.30564/jgm.v2i1.2242 
quality of life of patients with COPD. The SGRQ score is highly sensitive to determine changes in the condition of patients

\subsection{Nutritional Status Analysis}

Blood samples were collected in the early morning from 8:00 to 9:00 am after fasting for at least $11 \mathrm{~h}$. The levels of serum albumin (ALB), total protein (TP), hemoglobin $(\mathrm{Hb})$ were measured with a full-automatic biochemistry analyzer (7600-120 HITACHI, Japan). Body mass index (BMI) was calculated as weight (kilograms) divided by height (meters) squared.

\subsection{Statistical Analysis}

Data were analysed using IBM SPSS 22.Continuous data were expressed as means \pm standard deviation (SD). To compare continuous variables, an independent-sample $t$ test was performed. Results among three groups were evaluated by analysis of variance (ANOVN) using Scheffe's F test. The chi-squared test was used to analyze categorical data. P-values of less than 0.05 were consid-ered significant.

\section{Results}

There were no significant differences between Interventions and controls for TB, HB, $(\mathrm{P}>0.05)$. Compared with the control group, BMI, ALB, 6MWT,FVC, FEV1, FEV1/FVC and FEV1 as a percentage of expected value $\%$ levels were significantly higher in intervention group $(\mathrm{P}<0.05)$. Compared with the control group, Symptom scores, Activity score, Influence score and Total score were significantly lower in intervention group $(\mathrm{P}<0.05)$.

Table 1. Changes of nutritional indicators in the two groups

\begin{tabular}{|c|c|c|c|c|}
\hline & $\begin{array}{c}\text { Controls } \\
(\mathrm{n}=79)\end{array}$ & $\begin{array}{c}\text { Interventions } \\
(\mathrm{n}=76)\end{array}$ & $t / x^{2}$ & $P$ \\
\hline Age(year) & $75.6 \pm 3.5$ & $73.1 \pm 3.1$ & 1.421 & 0.167 \\
\hline $\mathrm{BMI}(\mathrm{kg} / \mathrm{m} 2)$ & $18.35 \pm 1.32$ & $21.10 \pm 1.90$ & 10.465 & 0.001 \\
\hline $\operatorname{ALB}(g / L)$ & $31.17 \pm 3.68$ & $34.3 \pm 2.54$ & 6.189 & 0.003 \\
\hline $\mathrm{TB}(\mathrm{g} / \mathrm{L})$ & $56.34 \pm 3.15$ & $57.05 \pm 3.67$ & 1.310 & 0.138 \\
\hline $\mathrm{Hb}(\mathrm{g} / \mathrm{L})$ & $125.02 \pm 10.91$ & $127.34 \pm 11.39$ & 1.302 & 0.416 \\
\hline $\mathrm{FVC}(\mathrm{L})$ & $2.10 \pm 0.18$ & $2.49 \pm 0.22$ & 11.999 & 0.001 \\
\hline FEV1 (L) & $1.02 \pm 0.12$ & $1.42 \pm 0.13$ & 6.189 & 0.000 \\
\hline FEV1/FVC & $49.58 \pm 4.46$ & $58.86 \pm 4.16$ & 13.418 & 0.002 \\
\hline FEV1 as a percentage of expected value $\%$ & $47.12 \pm 4.41$ & $58.83 \pm 4.22$ & 16.924 & 0.001 \\
\hline Symptom scores & $54.43 \pm 3.51$ & $44.23 \pm 4.73$ & 15.303 & 0.000 \\
\hline Activity score & $67.20 \pm 5.99$ & $60.81 \pm 5.79$ & 6.773 & 0.001 \\
\hline Influence score & $45.66 \pm 3.17$ & $33.31 \pm 3.67$ & 23.600 & 0.000 \\
\hline Total score & $58.53 \pm 4.16$ & $48.12 \pm 4.63$ & 14.766 & 0.001 \\
\hline 6MWT (m) & $198.25 \pm 8.58$ & $359.36 \pm 9.37$ & 111.962 & 0.000 \\
\hline
\end{tabular}

\section{Discussion}

COPD is a common disease among the elderly, with high morbidity and mortality. It seriously affects the quality of life of elderly patients, but also aggravates the economic burden of the family and society. Malnutrition has adverse effects on the structure and function of the body. Weak the body's resistance, and thus affec the clinical outcome of patients. Studies have shown that elderly COPD patients are often accompanied by malnutrition due to the deterioration of the functions of various organs in the body. The function of the digestive system, and the severity of COPD and its complications is significantly correlated with malnutrition ${ }^{[11-12]}$. Malnutrition not only reduces re- 
spiratory muscle function, but also weakens respiratory ventilatory reflex. By affecting the structure of lung tissue, it reduces the immune defense function. Weak its respiratory function. One of the independent risk factors for clinical adverse outcomes in elderly patients with COPD is malnutrition. Therefore, improving the nutritional status of elderly patients with COPD combined with malnutrition can significantly improve lung function. Significant implications for disease progression and quality of life. The 2018 revision of the Global Initiative on CHRONIC Obstructive Pulmonary Disease (GOLD) also makes it clear, that relieving symptoms, improving exercise endurance and halting disease progression were remain the main goals of COPD management worldwide.

Home enteral nutrition includes oral or tubular feedin. It is a way to provide energy and nutrients needed to patients through the gastrointestinal tract at home ${ }^{[13]}$. Oral enteral nutrient can avoid gastrointestinal mucosal atrophy, intestinal flora imbalance and intestinal bacteria and toxin diffusion ${ }^{[14]}$. It is a safe and effective method.

Nutrition mixed suspension can be the best choice. It is composed of water, maltodextrin, casein, vegetable oil, dietary fiber, minerals, vitamins and a variety of trace elements. It can provide the necessary nutrients for human body, especially in older patients. Oral can play a good nutrition effect ${ }^{[15]}$.

However, most of the current studies focus on nutritional support for hospitalized patients with COPD. Nutritional intervention for elderly patients with COPD at stable stage is obviously insufficien. There are few reports on nutritional intervention and less attention to the quality of life of discharged patients. Therefore, in this study, the changes in nutritional indicators, lung function, exercise tolerance and quality of life of the patients were studied. The results showed that compared with the diet alone, the enteral nutrition support not only improved the nutritional status of the patients, but also enhanced the lung function, activity tolerance and the quality of life of the patients. The results of our are study basically consistent with previous related studies ${ }^{[16]}$.

To sum up, early family enteral nutrition support can be a early,safe and effective adjunct to treatment for elderly patients with COPD with stable stage of malnutrition. This study focuses on the nutritional status of the elderly, pays attention to nutritional assessment. Carring out nutritional support as early as possible has significant prognostic significance for the disease. The shortcomings of this study are that the sample size of this study is small. Therefore, a larger sample size and a more rational design of multi-center, high-quality randomized controlled study are needed to further clarify the value of family enteral nutrition in elderly patients with COPD at stable stage.

\section{References}

[1] Geriatric nutrition support group, parenteral parenteral nutrition branch, Chinese medical association. Expert consensus on parenteral nutrition support for geriatric patients[J]. Chinese journal of geriatric medicine, 2013, 32(9): 913-929.

[2] Vogelmeier C F, Criner G J, Martinez F J, et al. Global strategy for the diagnosis, management, and prevention of chronic obstructive lung disease 2017 report. GOLD executive summary[J]. American journal of respiratory and critical care medicine, 2017, 195(5): 557-582.

[3] Rongchang Chen, Dongxing Zhao. The disease burden and patient characteristics of chronic obstructive pulmonary disease in China should be paid more attention[J]. Chin med med, 2017, 97(40): 3121-3123.

[4] Shurui Wang, Wenping Mao, Kiwu Huang. Chronic obstructive pulmonary disease: thinking and prospect $[\mathrm{J}]$. Chinese journal of multiorgan disease in the elderly, 2017,16(3): 164-168.

[5] Vogelmeier CF, Criner GJ, Martinez FJ, et al. Global Strategy for the Diagnosis, Management, and Prevention of Chronic Obstructive Lung Disease 2017 Report. GOLD Executive Summary. Am J Respir Crit Care Med., 2017, 195(5): 557-582. DOI: 10.1164/ rccm.201701-0218PP

[6] Chinese Medical Association, Chinese Medical Association Journal, Chinese Medical Association General Practice Branch, etc. Guidelines for primary diagnosis and treatment of chronic obstructive pulmonary disease (2018)[J]. Chinese Journal of General Practitioners, 2018, 17(11): 856.

[7] Ying Li, Yun Huang, Hongjuan Qian. Evaluation of nutritional status of hospitalized elderly patients by micro-nutrition evaluation method[J]. Chinese journal of clinical physicians (electronic edition), 2012, 06(4): 1026-1028.

[8] Lijuan Wang, Xi Yang, Xu Chen, Weiwei Yang, Junfei Zhu. Effects of oral nutrition supplementation on nutritional status and pulmonary function of elderly patients with chronic obstructive pulmonary disease discharged from hospital in a stable period[J]. Chinese Modern Doctors, 2008, 56(20): 119-122.

[9] Bernabeu-Mora R, Medina-Mirapeix F, Llamazares-Herrán $\mathrm{E}$, et al. The accuracy with which the 5 times sit-to-stand test, versus gait speed, can identify poor exercise tolerance in patients with COPD: A cross-sectional study[J]. Medicine, 2016, 95(35).

[10] Swigris J J, Esser D, Conoscenti C S, et al. The psychometric properties of the St George's Respiratory 
Questionnaire (SGRQ) in patients with idiopathic pulmonary fibrosis: a literature review[J]. Health and quality of life outcomes, 2014, 12(1): 124.

[11] Terashima T, Chubachi S, Matsuzaki T, et al. The association between dental health and nutritional status in chronic obstructive pulmonary disease[J]. Chronic respiratory disease, 2017, 14(4): 334-341.

[12] Terashima T, Chubachi S, Matsuzaki T, et al. The association between dental health and nutritional status inchronic obstructive pulmonary disease[J]. Chron Respir Dis, 2017, 14 (4): 334-341.

[13] Matkovic Z, Cvetko D, Rahelic D, et al. Nutritional status of patients with chronic obstructive pulmonary disease in relation to their physical performance[J]. COPD, 2017, 14(6): 626-634.

[14] Chen lingzhi, Ming jingjing, li qing, et al. Nutrition evaluation and application in patients with chronic obstructive pulmonary disease[J]. Chinese journal of lung diseases (electronic edition), 2015,8 (5): 96-99.

[15] Relph WL. Addressing the nutritional needs of older patients[J]. Nurs Older People, 2016, 28 (3): 16-19.

[16] Elia M, Parsons E L, Cawood A L, et al. Cost-effectiveness of oral nutritional supplements in older malnourished care home residents[J]. Clinical Nutrition, 2018, 37(2): 651-658. 